\title{
Estado actual, costos económicos y sociales del pie diabético y las amputaciones en la población mexicana
}

\section{The economic and social costs of the diabetic foot and its amputations. Current situation in the Mexican population}

\author{
Adriana Torres-Machorro ${ }^{1}$, Christopher Ruben-Castillo², José F. Torres-Roldán ${ }^{3}$, \\ Óscar Francisco Miranda-Gómez ${ }^{1}$, Jorge Catrip-Torres ${ }^{1}$ y Carlos A. Hinojosa2*
}

${ }^{1}$ Servicio de Cirugía Vascular y Cardiotorácica, Instituto Nacional de Cardiología Ignacio Chávez; ${ }^{2}$ Servicio de Angiología y Cirugía Vascular, Instituto Nacional de Ciencias Médicas y Nutrición Salvador Zubirán; ${ }^{3}$ Colegio Mexicano de Ortopedia A.C. Ciudad de México, México

\begin{abstract}
Resumen
En los países industrializados, los altos costos del tratamiento de la diabetes mellitus derivan de las fallas en el proceso de respuesta a la enfermedad, sin embargo, en naciones emergentes, los elevados costos se asocian principalmente a la atención tardía de los pacientes. Los montos del tratamiento del pie diabético en México corroboran que existe una deficiencia en el sistema de salud. Por ello, los esfuerzos deben enfocarse a la prevención, pues el costo de un paciente con diabetes con buen control y sin complicaciones es de 8,000 pesos mexicanos anuales. Esta suma representa «poco» comparado con el costo del manejo anual de un solo paciente con complicaciones, que puede sobrepasar los 300.000 pesos, o incluso multiplicarse si se realiza una amputación mayor. Se debe fomentar una consciencia profunda de las catastróficas secuelas del pie diabético y, con ello, limitar la progresión de esta enfermedad. Es de extrema importancia modificar las imperfecciones en la atención médica e implementar estrategias preventivas eficaces a nivel local y nacional para minimizar la realización de amputaciones mayores y abatir sus consecuentes costos. La pérdida de una extremidad afecta al paciente y a la familia del enfermo. En estos casos, todos participamos de manera directa o indirecta en la reinserción de los enfermos a la vida productiva o normal, lo cual es imposible de lograr una vez que la extremidad se compromete seriamente, pues se afecta irreversiblemente el entorno del enfermo y a la sociedad en la que se desarrolla.
\end{abstract}

Palabras clave: Pie diabético. Costo económico. Costo social. Incapacidad. Amputación. Estrategia de prevención.

\section{Abstract}

The high costs derived from the diabetes management in industrialized countries ar the result from failure in the disease response process, contrasting with those in developing nations, where late disease treatment increases the costs. The expenses of diabetic foot treatment in Mexico confirm that the health system is deficient. Therefore, efforts should be focused on prevention, since the spending of a patient with well-controlled and uncomplicated diabetes is 8,000 Mexican pesos per year. This sum represents a small amount of money compared to the one of managing a single patient with complications,

Correspondencia:

${ }^{*}$ Carlos A. Hinojosa

E-mail: carlos.a.hinojosa@gmail.com
Disponible en internet: 21-07-2020

Rev Mex Angiol. 2020;48(2):53-64

www.RMAngiologia.com

0377-4740/C 2020 Sociedad Mexicana de Angiología y Cirugía Vascular y Endovascular, A.C. Published by Permanyer México. This is an open access article under the CC BY-NC-ND license (http://creativecommons.org/licenses/by-nc-nd/4.0/). 
which may exceed 300,000 Mexican pesos per year or more if amputation is performed. The deleterious effects of the diabetic foot must foster a deep awareness and thereby help to prevent disease' progression. It is essential to modify the imperfections in medical care and to apply effective prevention strategies, locally and nationally, to reduce the amount of above the knee amputations and lower their burden. Limb loss concerns not only the patient but the patient's family. In these cases, we all participate directly or indirectly to reintegrate patients into a productive and active life. This task becomes almost impossible once the limb is seriously affected because it irreversibly changes the patient's environment and the society in which he or she interacts.

Key words: Diabetic foot. Economic burden. Social burden. Handicap. Amputation. Prevention strategy.

\section{Introducción}

México ocupa el quinto lugar en prevalencia de diabetes mellitus (DM) a nivel global. La Federación Internacional de Diabetes calculó en 2017 que la población mexicana con DM es superior a 12 millones, con una prevalencia del $13.6 \%$ en adultos de 20 a 79 años, y estima que para el año 2045 será de 21.8 millones, aproximadamente 1 . Las complicaciones de la DM son resultado de la toxicidad ligada a estados prolongados de hiperglucemia. Estas complicaciones afectan al paciente, a su familia y a la sociedad, al enfrentar altas tasas de incapacidad, pérdida de autonomía y mortalidad.

La prevalencia de úlcera por pie diabético va del 1\% en países europeos hasta el $11 \%$ en países del continente africano ${ }^{2}$. Esta variabilidad tiene una relación directa con factores sociales, económicos y los intrínsecos a la salud de cada paciente. En México, en el año 2012 se reportó que el $47.6 \%$ de pacientes con diabetes referían ardor, dolor o pérdida de sensibilidad en los pies. El $7.2 \%$ presentaba úlceras en las piernas o en los pies y el $2 \%$ de los pacientes tenían una amputación ${ }^{3}$. Esta úlcera se desarrollará en la cuarta parte de las personas con DM en el transcurso de sus vidas y es igualmente un signo ominoso, pues precede al $80 \%$ de las amputaciones por pie diabético. Además de la falta de prevención, existen factores que determinan la amputación, como la infección y la enfermedad arterial periférica (EAP), y otros que la propician, es decir, neuropatías, deformidad significativa del pie, rango de movimiento disminuido, presiones plantares elevadas, traumatismo menor, úlcera o amputación previa y alteraciones visuales. Patologías que justifican un abordaje multidisciplinario ${ }^{4}$. Por consiguiente, el tratamiento de las secuelas es comparativamente mayor a los costos de prevención. Se debe, entonces, transformar la mentalidad hacia la revascularización como primera intención de tratamiento. El cirujano vascular tiene un papel destacado al revascularizar, pues, limita el nivel de amputación o incluso puede descartarla. El nivel de amputación incide en la calidad de vida, la reincorporación del paciente a la sociedad y su riesgo de mortalidad postoperatoria. En amputaciones por debajo de la rodilla, entre el 50 y el $100 \%$ de los pacientes usa prótesis, mientras que su uso en aquellos pacientes con una amputación por arriba de la rodilla disminuye entre el 10 y el $30 \%$; es decir, utilizan muletas o andadera, que ponen en riesgo los tendones del mango rotador del hombro, lo que los lleva a experimentar una mayor discapacidad. En ausencia de un protocolo establecido, la selección de nivel puede ser equivocada y secundar múltiples cirugías para asegurar una adecuada vascularización del muñón hasta en el 15\% de los casos 5 .

Además, se calcula que solo una de cada diez personas con una extremidad amputada se rehabilita, y que el $30 \%$ de estos sabe usar de forma adecuada las órtesis indicadas. Infortunadamente, el costo de estos dispositivos (aproximadamente 100.000 pesos) restringe su adquisición ${ }^{6}$, aunque existen prótesis mucho más costosas.

Recordar que entre un 15 y un $25 \%$ de los casos serán eventualmente candidatos a una amputación contralateral ${ }^{7}$. Una vez que un individuo pierde las dos extremidades, su capacidad de deambulación y productividad disminuyen drásticamente, y necesitará de cuidados institucionales o familiares. Los pacientes se verán envueltos en una serie de complicaciones que conferirán mayores costos a su propia economía y al sistema de salud.

La mortalidad en pacientes con pie diabético se duplica en comparación con pacientes que no sufren esta complicación, y al amputarse se registra una mortalidad a 5 años del $70 \%^{8}$. Es trágico que alrededor del $1 \%$ de los pacientes con DM será amputado. Si consideramos que la DM es responsable del 25 y el $90 \%$ de las amputaciones no traumáticas, las mismas que ocurren cada 20 segundos, esto equivale a cerca de un millón de personas amputadas al año ${ }^{9}$.

Es importante conocer los costos y el verdadero impacto económico de la dm para implementar estrategias 
que reduzcan su morbimortalidad en México. El análisis económico debe tomar en cuenta el impacto en la productividad de los mexicanos diabéticos y la carga económica que representan. Debe considerar el costo directo en gastos de la atención médica y el costo indirecto; es decir, el efecto de una mortalidad prematura y la capacidad de una persona para desempeñarse en el aspecto laboral (ausentismo, incapacidad e invalidez, disminución del ingreso familiar y de la contribución a la producción del país). Situación que se extiende al nicho familiar y/o a las personas dedicadas al cuidado de los enfermos.

\section{Costos de la atención del pie diabético y las amputaciones}

En el 2013, los costos de la DM en México se estimaron en $362,859.82$ pesos, equivalentes al $2.25 \%$ del producto interno bruto (PIB). En cuanto a costos directos, se estimaron en $179,495.3$ pesos, equivalentes al $1.1 \%$ del PIB; en el cual, el $87 \%$ derivó de la atención de las complicaciones. Los costos indirectos secundarios a la pérdida de ingresos y productividad por muerte prematura y discapacidad se calcularon en 183,364.49 pesos $(1.14 \% \text { del PIB })^{10}$ (Tabla 1).

Barraza Llorens, et al. ${ }^{10}$ calcularon un costo anual por evento de úlceras en los pies de 1,011 pesos en 2013. Cuando este valor se compara con los costos de una amputación, los resultados son inadmisibles, porque se estiman en 85,428 pesos por evento, así como los costos totales de la atención médica de las amputaciones, que alcanzan 9,873.4 millones de pesos. A pesar de que no toman en cuenta los costos indirectos, aun estas cifras proyectan la gravedad de las complicaciones y evolución natural del pie diabético.

Los costos son cubiertos en su mayor proporción por el Instituto Mexicano del Seguro Social (IMSS), que asume el $38 \%$ del total, seguido por el medio privado, con el $25 \%$, y la Secretaría de Salud (SS), con el $23 \%$, que comprende los servicios estatales de salud, los institutos nacionales de salud, los hospitales federales de referencia, los regionales de alta especialidad y en su momento el Seguro Popular ${ }^{10}$. No obstante, en México, el $49.4 \%$ del gasto total en salud proviene del sector privado, independientemente de que exista alguna afiliación a un sistema público de salud, lo cual plantea una situación grave con perspectiva de costos indirectos en los hogares ${ }^{11}$, pues obliga a reducir gastos en otros bienes o servicios, adquirir deudas y/o modificar patrones de consumo e inversión.
Tabla 1. Carga económica de la diabetes mellitus en México: costos indirectos (millones de pesos de 2013 y PIB) \begin{tabular}{l|l} 
Rubro & Costo en millones de pesos (\% PIB)
\end{tabular}

\begin{tabular}{|l|c|}
\hline Muerte prematura & $132,990.90(0.83)$ \\
\hline Ausentismo laboral & $689.97(0.004)$ \\
\hline Incapacidad laboral & $16,361.40(0.10)$ \\
\hline Invalidez & $16,571.74(0.10)$ \\
\hline Presentismo & $16,750.48(0.10)$ \\
\hline Total & $183,364.49(1.14)$ \\
\hline
\end{tabular}

PIB: producto interno bruto. Modificado de Ragnarson Tennvall G, et al. ${ }^{16}$.

En este sentido, el IMSS reporta un ausentismo por úlceras por pie diabético de un promedio de 2,433 días autorizados y 11,920 días de incapacidad ${ }^{12}$. Las amputaciones reportaron un ausentismo de 2,472 días y 19,833 días de incapacidad, solo por debajo de casos de infarto agudo al miocardio (28,000 días) y nefropatía (19,868 días). El costo del ausentismo laboral se estima en 689.9 millones de pesos (0.4\%), la incapacidad laboral en 16,361.4 millones de pesos y la invalidez en $16,571.7$ millones de pesos $(9.0 \%)^{10}$.

En otro análisis ${ }^{13}$, se estudiaron costos directos con base en la gravedad de la úlcera por pie diabético. El costo más alto fue para la clasificación Wagner 5 , incluyendo estudios de laboratorio (924 pesos), rayos $X$ (798 pesos), amputaciones mayores (14,871 pesos) e interconsultas por especialidad (2,305 pesos). El análisis destaca que aquellos con clasificación Wagner 4 reportaron costos promedio más altos por día-cama (50,780 pesos) y debridaciones (14,871 pesos), incluso mayores que Wagner 5 . Estos autores concluyen que los costos se incrementan proporcionalmente a la gravedad de la enfermedad. Resulta interesante ubicar que, de 1990 a 2010, la DM en México conformó la décima causa de años de vida perdidos por muerte prematura en hombres, con 280,985 años de vida perdidos, y la quinta causa en mujeres, con 324,988 años de vida perdidos. Los años de vida perdidos por muerte prematura, al igual que los años de vida asociados a discapacidad, integran el indicador de años de vida saludable perdidos (AVISA). En el análisis de las principales causas de años vividos asociados a discapacidad, en 2010, la DM fue la quinta causa, con 275,224 años. En esta misma observación, la DM debutó como la primera causa de AVISA perdidos, con 801,948 años de vida perdidos ${ }^{14}$. Se calculan, además, costos 
Tabla 2. AVISA por complicaciones prevenibles de DM en México (2001-2011)

\begin{tabular}{|l|c|c|}
\hline Complicaciones & Total AVISA & Porcentaje de AVISA (\%) \\
\hline Falla renal & 366,808 & 63 \\
\hline Retinopatía & 34,774 & 6 \\
\hline Neuropatía & 18,051 & 3 \\
\hline Pie diabético & 60,750 & 10 \\
\hline Amputación & 102,233 & 18 \\
\hline Total & 582,616 & \\
\hline
\end{tabular}

AVISA: años de vida saludable perdidos; DM: diabetes mellitus.

indirectos por 183,364.4 millones de pesos, en donde la mayor proporción corresponde a muerte prematura, calculados en $132,990.9$ millones de pesos $(72.5 \%)^{10}$ (Tabla 2).

La carga económica total de la DM equivale a 7,700 millones de dólares (MDD), de los cuales, 3,400 millones de dólares corresponden a costos directos y 4,300 a costos indirectos ${ }^{15}$. La mayor proporción es cubierta por los pacientes (4,154 millones de dólares), seguido del IMSS (2,084 millones de dólares), la SS (909 millones de dólares) y, por último, las aseguradoras privadas (103 millones de dólares). El desarrollo de índices económicos que permiten la distribución de recursos basada en criterios de efectividad con respecto a rubros clínicos, epidemiológicos, económicos y administrativos se basa en estos datos.

Este problema atañe también al empleador, pues su participación conforma el $15.1 \%$ del total de los costos indirectos totales. Con base en dictámenes de invalidez expedidos por el IMSS, el componente de ausentismo laboral se calculó en 8.94 millones de pesos (1.9\%), incapacidad laboral en 295.43 millones de pesos $(61.4 \%)$ e invalidez en 176.82 millones de pesos ${ }^{10}$. En virtud de lo antes expuesto, las discrepancias en salud surgidas por la carga económica de la diabetes sugieren la reestructuración de sistemas de salud para evolucionar los modelos curativos hacia los preventivos, con el objetivo de vencer los grandes desafíos que impone esta enfermedad.

\section{Estrategias costo-efectivas en el tratamiento del pie diabético aplicables en México}

El modelo predictivo de Markov es frecuentemente utilizado para determinar el beneficio económico de
Tabla 3. Equipo multidisciplinario en la valoración del enfermo con pie diabético

\begin{tabular}{l}
$\begin{array}{l}\text { Angiología y cirugía vascular, la cual tiene un papel } \\
\text { predominante en el salvamento de la extremidad }\end{array}$ \\
\hline Endocrinología (diabetología) \\
\hline Cirugía de pie (ortopedia y cirugía plástica) \\
\hline Especialista en heridas \\
\hline Nutriología clínica \\
\hline Radiología intervencionista \\
\hline Ortesista \\
\hline Rehabilitación \\
\hline Médico general
\end{tabular}

una intervención realizada, pues toma en cuenta la cronicidad de la enfermedad y las recurrencias del mismo evento (p. ej., úlcera infectada por pie diabético $)^{16}$. Sin embargo, su aplicabilidad en distintos estudios en nuestro país se dificulta debido a las diferencias demográficas y en los sistemas de salud. A pesar de estas limitaciones, el tratamiento multidisciplinario está considerado como la mejor estrategia a implementar en México, pues a nivel mundial disminuye y/o previene las amputaciones asociadas al pie diabético. Esta información se contrasta con el manejo quirúrgico o médico que, de forma aislada, falla en el salvamento de la extremidad ${ }^{17}$.

El equipo multidisciplinario involucra una asesoría clínica y vascular estructurada derivada del compromiso de múltiples especialidades (Tabla 3).

Gracias a la toma de decisiones colegiada con criterios uniformes al elegir a quiénes se debe hospitalizar, intervenir o realizar estudios de extensión, el manejo multidisciplinario logra la reducción del $78 \%$ en las amputaciones mayores en pie diabético ${ }^{18}$. Estos resultados disminuyen la estancia hospitalaria y minimizan el costo total del manejo de la enfermedad ${ }^{17}$.

La reducción anual de costos se estima de 1,100,000 dólares, 750,000 dólares y 850,000 dólares por cada una de las siguientes intervenciones realizadas: la educación preventiva, el manejo por equipo multidisciplinario y la utilización de un calzado adaptado, respectivamente. Además, se disminuye la cantidad de amputaciones en el 72,47 y $53 \%$, respectivamente ${ }^{19}$. De hecho, estas medidas se traducen en un ahorro de entre 2,900 y 4,442 dólares por paciente, en cada tratamiento. El conjunto de especialidades que integran este equipo 
se encuentra disponible, vigente y altamente capacitado en nuestro entorno. La concentración en centros de referencia facilita el acceso y el apego del paciente, sin embargo, hay que complementar con equipos móviles para aquellos con limitaciones (físicas, sociales y/o económicas) de acceso al servicio de salud integral. El enfoque específico en esta patología favorece el tratamiento óptimo y disminuye los posibles errores en la toma de decisiones $^{20}$.

Los esfuerzos del equipo multidisciplinario deben enfocarse en la educación. Tanto el enfermo como su familia deben ser capacitados para la prevención del pie diabético con intención de mejorar la calidad de vida y disminuir la cantidad de amputaciones mayores realizadas. La toma de decisiones debe acompañarse de la educación y actualización continua de los prestadores de servicio, de los médicos de primer contacto y de la población general, ya que favorece la referencia, el diagnóstico temprano y la limitación de complicaciones ${ }^{21}$. Como consecuencia directa habrá un abatimiento del costo del manejo de las complicaciones de una úlcera ${ }^{16}$. Los índices costo-efectividad con resultados más favorables estarán fuertemente asociados a estrategias de prevención de la aparición de úlceras por pie diabético ${ }^{22}$.

Es recomendable realizar un manejo integral de esta patología con base en guías o protocolos con evidencia científica. Al aplicar el consenso del International Working Group on the Diabetic Foot ${ }^{23}$ se han disminuido las amputaciones mayores. Se justifica, por lo tanto, el desarrollo de un consenso mexicano para el manejo del pie diabético. Por último, es importante destacar que los efectos benéficos y el costo-efectividad de esta estrategia no son efímeros, sino que permanecen por largo tiempo ${ }^{24}$.

\section{La Estrategia Nacional de Prevención y otras iniciativas comerciales complementarias}

A partir del año 2013, debido al crecimiento sostenido de la diabetes y sus complicaciones, el gobierno mexicano implementó la Estrategia Nacional para la Prevención y Control del Sobrepeso, la Obesidad y la Diabetes ${ }^{25}$. Esta decisión se tomó debido a los exorbitantes gastos que representa tal enfermedad y a la pérdida catastrófica en AVISA, pues ocasionó el $13 \%$ de las defunciones en el año $2011^{26}$ y el $15.2 \%$, en 2017, lo que la colocó en el primer y segundo lugar, respectivamente, entre las principales causas de mortalidad en México ${ }^{27}$. A pesar de los esfuerzos realizados, únicamente el $25 \%$ de la población tiene un control metabólico aceptable, por lo que aún no es visible una limitación de la progresión de la enfermedad ni en la ocurrencia de complicaciones ${ }^{28}$. Esta estrategia intenta mejorar el control de los pacientes diabéticos y promueve una política nacional que genere hábitos de consumo alimenticio y actividad física saludables. Su finalidad es aminorar el riesgo de mortalidad prematura por diabetes involucrando a la sociedad civil y a los sectores público y privado. Se busca, además, un diagnóstico genético y detener el incremento en sobrepeso, obesidad y diabetes. Las estrategias deben organizarse alrededor de sus tres pilares $^{29}$ :

1. La Salud Pública. Abarca la promoción de la salud y la educación preventiva con énfasis en la realización de actividad física. Incluye el mejor control metabólico obtenido al promover el deporte, la educación de salud centrada en el empoderamiento del paciente a través de facilitadores en educación en DM, herramientas de educación disponibles en línea al público en general y la disminución drástica de la promoción de alimentos industrializados. La realización aislada de alguna de estas medidas disminuye exponencialmente el impacto en la población, al igual que para aquellos que no tienen acceso a la difusión por medios electrónicos.

2. La atención médica. Incluye el acceso efectivo a los servicios de salud a través de centros de atención del paciente diabético, modelos integrales de atención médica e investigación genómica. Este rubro se explica con ejemplos como el Centro de Atención Integral del Paciente con Diabetes (CAIPaDi), que es resultado del esfuerzo de la Federación Mexicana de Diabetes A.C. y del Instituto Nacional de Ciencias Médicas y Nutrición Salvador Zubirán (INCMNSZ). Este programa se caracteriza por realizar a lo largo de un día múltiples intervenciones médicas y paramédicas en pacientes con DM de menos de 5 años de evolución. Las sesiones son mensuales durante un periodo de cuatro meses y continúa con una vigilancia remota al terminar el programa. Este modelo ha demostrado una mejoría y mantenimiento sostenido de las metas de tratamiento al mantener la $\mathrm{He}$ moglobina glicosilada $(\mathrm{HbA} 1 \mathrm{c})<7$ en el $61 \%$, una tensión arterial < 130/80 mmHg en el $70.6 \%$ y un colestero lipoproteínas de baja densidad (LDL) < $100 \mathrm{mg} / \mathrm{dl}$ en el $40.8 \%$ de los pacientes posterior a 2 años $^{30}$. Independientemente a lo propuesto por la Estrategia Nacional, el IMSS cuenta con el programa DiabetIMSS, que establece una estrategia 


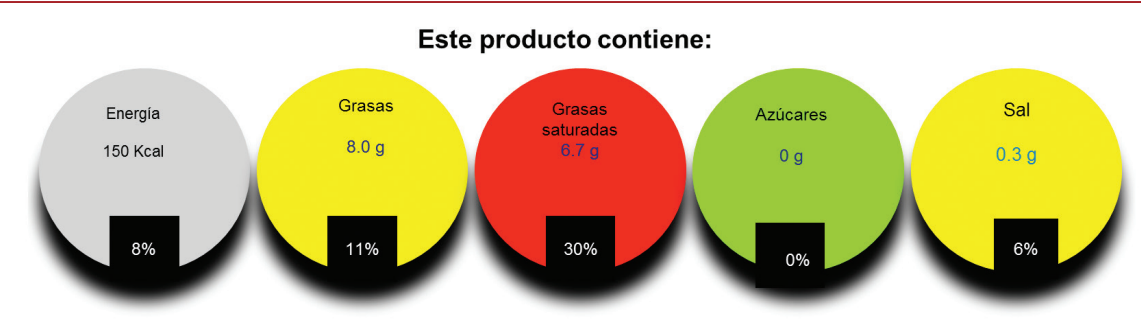

*Porcentaje del valor diario recomendado

Figura 1. Ejemplo de etiquetado frontal en alimentos siguiendo el modelo del semáforo.

multidisciplinaria en el primer nivel de atención. A lo largo de 1 año inculca en el paciente la reflexión y la toma de decisiones informada mediante consultas médicas y sesiones educativas mensuales. Su ejecución ha demostrado un efecto significativo al lograr un mejor control metabólico y cambios en el estilo de vida en el $71 \%$ de los pacientes que completaron su instrucción, comparados con aquellos que se encontraban aún en formación o que no recibieron formación alguna (32 y 17.2\%, respectivamente) ${ }^{31}$. Ambos programas contemplan el manejo de otras patologías asociadas, como el tabaquismo y el alcoholismo. Sin embargo, a pesar de las grandes ventajas que demuestran, la mayor parte de la población no tiene acceso a esta atención preventiva y presentará alguna complicación. Es por esto que modelos como estos deben replicarse e instalarse en forma rápida en nuestra población.

3. La regulación sanitaria. Regula el etiquetado y la publicidad de alimentos y bebidas, en especial aquellas dirigidas al público infantil. Propone el etiquetado frontal obligatorio, la división de productos en categorías claras y de fácil entendimiento, el establecimiento de horarios de audiencia infantil con limitación de publicidad, y la aplicación de un impuesto a alimentos y bebidas de escaso contenido nutricional $^{32}$ (Fig. 1).

Es primordial el compromiso de los pacientes diabéticos y de su entorno para el éxito de cualquier intervención. La salud es una responsabilidad compartida exitosa bajo condición de participación activa de las tres órdenes de gobierno, la sociedad civil y las organizaciones no gubernamentales, además de las academias, de la Fundación Mexicana para la Salud y del sector privado ${ }^{25}$. Es correcto enfocarse principalmente en la prevención de la diabetes a fin de intentar coartar sus efectos educando desde la infancia. Sin embargo, no existen políticas específicas de manejo y/o prevención secundaria del pie diabético que sean accesibles a la población general en temas de educación y orientación médica especializada. Resulta lógico que los esfuerzos deban dirigirse a la prevención primaria, no obstante, dado que, en la actualidad, las úlceras por pie diabético y las amputaciones se encuentran entre las complicaciones más reportadas, y que el $46.4 \%$ de la población encuestada no realiza medidas preventivas para evitar o retrasar estas complicaciones, deben instaurarse también con urgencia políticas de prevención secundaria ${ }^{33}$.

\section{Marco jurídico y Cuadro básico nacional para el tratamiento del pie diabético}

Las normativas con potencial impacto en el tratamiento de la DM son determinadas por el Consejo de Salubridad General (CSG), que depende en forma directa del Presidente de la República ${ }^{34}$. Corresponde también al CSG, liderado por el Secretario de Salud, la gestión de los trabajos de la Comisión Interinstitucional del Cuadro básico y Catálogo de insumos del sector salud. Como tal, no existe un Cuadro básico para la prevención del pie diabético, pero dentro de cada uno se encuentran repartidos múltiples elementos para lograr su diagnóstico, control y tratamiento integral.

El Cuadro básico propone materiales que buscan ser de buena calidad, estar autorizados por la Cofepris y la SS y tener un precio accesible. Se busca cumplir con los requerimientos mínimos necesarios para garantizar la prestación de servicios, estandarizar su uso y favorecer su adecuada prescripción. Asimismo, intenta impedir el uso de medicamentos peligrosos y, de manera secundaria, controlar los gastos. Esto no significa que los medicamentos se encuentren disponibles en todos los servicios de salud de México, ya que cada 
uno realiza su selección mediante comités institucionales que toman en cuenta el presupuesto asignado a cada hospital y las necesidades de acuerdo al nivel de atención. Este presupuesto es determinado por los recursos asignados por el gobierno federal a todas las instituciones públicas, excepto al IMSS, en el que el gobierno solo participa en una parte del mismo.

Los cuadros básicos derivados de la Comisión Interinstitucionales en 2017 fueron realizados con la participación de la SSA, el IMSS, el Instituto de Seguridad y Servicios Sociales de los Trabajadores del Estado (ISSSTE), la Secretaría de Marina (SEMAR), la Secretaría de la Defensa Nacional (SEDENA) y Petróleos Mexicanos (PEMEX). A pesar de que esto representó un esfuerzo institucional positivo, una vez entendida la variación de acuerdo a la selección de cada comité, resulta fácil comprender las diferencias que existen entre los cuadros básicos de cada institución.

La desvinculación entre los diferentes manuales dificulta el manejo integral de esta patología. En ocasiones, estas propuestas se han trabajado en distintos momentos con diferentes tendencias, por lo que homologar la práctica médica se vuelve una tarea casi imposible. Es por ello que los consensos con los comités interinstitucionales ameritan actualización continua e integrarse por personas altamente capacitadas en la atención de un paciente con pie diabético. Además, se debe de vigilar la justa aplicación y distribución de los productos y servicios.

En la tabla 4 se detallan los agentes considerados como útiles para el diagnóstico, el establecimiento de pie diabético, con o sin enfermedad vascular asociada, el control metabólico de la diabetes y enfermedades asociadas. Se toman en cuenta los posibles participantes en caso de sobreinfección, el tratamiento quirúrgico no solo enfocado en la amputación sino en la revascularización, manejo y curación de heridas secundarias al pie diabético. Por último, se consideran los esfuerzos en rehabilitación posterior a amputación y otras herramientas fuera del Cuadro básico, que afinan el manejo ${ }^{34-38}$ (Tabla 4).

La inclusión de estos medicamentos, insumos y materiales dentro del Cuadro básico determina que el pie diabético se defina como una enfermedad que provoca gastos catastróficos. Además, las regulaciones técnicas de carácter obligatorio que buscan garantizar los requisitos mínimos de información, seguridad, calidad y servicio se establecen en la Norma Oficial Mexicana para la Prevención, Tratamiento y Control de la Diabetes ${ }^{39}$, la cual fue redactada en 2010. Su formulación es responsabilidad de la Secretaría de Salud y en el inciso
11.11.4 estipula recomendaciones básicas para el pie diabético.

Aciertos de esta norma son tanto el cuidado en el calzado como la inspección periódica de los pies. Asimismo, se contempla la valoración por un especialista en caso de detectar neuropatía y el envío inmediato al especialista en caso de infección. También se recomienda utilizar aspirina en dosis bajas y la referencia a segundo nivel o al especialista en caso de complicaciones clasificadas como graves (insuficiencia arterial de miembros inferiores, lesiones isquémicas o neuropáticas de miembros inferiores, neuropatías diabéticas de difícil control o las infecciones frecuentes). Por desgracia, estos casos denotan etapas tardías de la enfermedad, donde el impacto en la calidad de vida ya está comprometido y el salvamento de la extremidad es menor.

Deberá considerarse, en la actualización de esta norma, la inclusión del pie diabético como complicación grave, la precisión del especialista de referencia o la atención multidisciplinaria y la individualización del calzado adaptado a la anatomía, los puntos de presión, y las modificaciones posteriores a una amputación. Por último, las Guías de práctica clínica para el manejo del pie diabético ${ }^{40}$ buscan homologar la práctica en segundo nivel. Plantean un manejo multidisciplinario para el diagnóstico, tratamiento metabólico, tratamiento quirúrgico, reconstrucción y rehabilitación. La inclusión de la revascularización endovascular y la actualización en el manejo abierto de las heridas con las terapias adyuvantes que propone logrará mejoras radicales en el tratamiento integral que plantea. Su limitación se encuentra en la disponibilidad exclusiva de algunos insumos e instalaciones en el tercer nivel de atención.

\section{Cobertura del pie diabético en la seguridad pública y en la privada}

\section{Cobertura en servicios de salud públicos}

\section{Secretaría de Salud}

Atiende al $26.8 \%$ de los diabéticos ${ }^{41}$. Existe una cobertura parcial para el tratamiento del pie diabético debido a que no se cuenta con los recursos humanos ni hay insumos disponibles en todas las instituciones de segundo nivel.

Una vez instalado el problema del pie diabético, su atención integral exige la participación de múltiples instituciones para acceder a un manejo multidisciplinario que culmine en la revascularización, la cual no está cubierta en su totalidad por este servicio. Sin embargo, 
Tabla 4. Medicamentos, insumos y equipo dentro del Cuadro básico de uso potencial para el manejo integral del pie diabético

\begin{tabular}{|c|c|c|c|}
\hline Nivel de impacto & Diagnóstico & Tratamiento médico & Tratamiento quirúrgico \\
\hline $\begin{array}{l}\text { Cuadro básico y } \\
\text { Catálogo de } \\
\text { medicamentos }\end{array}$ & & $\begin{array}{l}\text { Cardiología: AAS, pentoxifilina, } \\
\text { cilostazol, estatinas, IECA, ARA y } \\
\text { antagonistas de la vitamina K. } \\
\text { Hematología: nuevos ACO } \\
\text { (dabigatrán, apixabán, rivaroxabán), } \\
\text { heparina, enoxaparina, } \\
\text { fondaparinux, tinzaparina, } \\
\text { ticagrelor. } \\
\text { Endocrinología: insulina, } \\
\text { metformina, estatinas, gliptinas, } \\
\text { bezafibrato. } \\
\text { Enfermedades infecciosas y } \\
\text { parasitarias: múltiples antibióticos. }\end{array}$ & $\begin{array}{l}\text { Cardiología: dinitrato y mononitrato de } \\
\text { isosorbida. } \\
\text { Hematología: heparinas. }\end{array}$ \\
\hline $\begin{array}{l}\text { Cuadro básico y } \\
\text { Catálogo de } \\
\text { instrumental y } \\
\text { equipo médico }\end{array}$ & & & $\begin{array}{l}\text { Cirugía cardíaca (injertos): poliéster y } \\
\text { dacrón recubiertos de albúmina y } \\
\text { colágeno, anillados y bifurcados. } \\
\text { Microcirugía: pinza vascular DeBakey } \\
\text { Porta aguja especial: castroviejo con y sin } \\
\text { retén (para oftalmología). } \\
\text { Porta aguja para cirugía vascular. } \\
\text { Separador quirúrgico articulado. } \\
\text { Tijera cardiovascular. }\end{array}$ \\
\hline $\begin{array}{l}\text { Cuadro básico y } \\
\text { Catálogo de } \\
\text { material de } \\
\text { curación }\end{array}$ & $\begin{array}{l}\text { Cardiología de uso } \\
\text { general: aguja para } \\
\text { punción de vasos venosos } \\
\text { o arteriales, cánulas, } \\
\text { catéteres, cuerdas, guías } \\
\text { e introductores de } \\
\text { múltiples diámetros y } \\
\text { conformaciones. }\end{array}$ & $\begin{array}{l}\text { Médicas y quirúrgicas: gasas, } \\
\text { guantes, vendas elásticas. } \\
\text { Traumatología y ortopedia: férulas. }\end{array}$ & $\begin{array}{l}\text { Cardiología intervencionista: agujas, } \\
\text { catéteres, cuerdas, dilatadores, catéter } \\
\text { balón para angioplastia percutánea } \\
\text { transluminar de vasos periféricos } \\
\text { infrapoplíteos y femorales. } \\
\text { Injertos: de politretrafluoroetileno } \\
\text { anillados, injerto vascular de poliéster con } \\
\text { recubrimiento antibacteriano. } \\
\text { Suturas: sintéticas no absorbibles, } \\
\text { monofilamento de polipropileno, con } \\
\text { agujas dobles armadas de múltiples tallas. } \\
\text { Médicas y quirúrgicas: antisépticos, } \\
\text { apósitos. }\end{array}$ \\
\hline $\begin{array}{l}\text { Cuadro básico y } \\
\text { Catálogo de } \\
\text { auxiliares de } \\
\text { diagnóstico }\end{array}$ & $\begin{array}{l}\text { Anatomía patológica: } \\
\text { microscopios, } \\
\text { microtomos. } \\
\text { Agentes de diagnóstico: } \\
\text { medios de cultivo. } \\
\text { Química diagnóstica: } \\
\text { reactivos químicos, } \\
\text { analizadores, etc. } \\
\text { Radiodiagnóstico: medios } \\
\text { de contraste } \\
\text { Microbiología: cultivos. }\end{array}$ & & \\
\hline $\begin{array}{l}\text { Fuera de Cuadro } \\
\text { básico }\end{array}$ & $\begin{array}{l}\text { Doppler vascular continuo } \\
\text { portátil de } 5 \text { u } 8 \mathrm{mHz} \text { para } \\
\text { medición de índice } \\
\text { tobillo-brazo. } \\
\text { Medición de presión } \\
\text { transcutánea de oxígeno. } \\
\text { Medición del índice } \\
\text { tobillo- dedo. } \\
\text { Monofilamento. }\end{array}$ & $\begin{array}{l}\text { Sistema adyuvante de cierre por } \\
\text { presión negativa. } \\
\text { Prótesis u ortésis para } \\
\text { rehabilitación posterior a una } \\
\text { amputación del miembro inferior. }\end{array}$ & Valvulotomo. \\
\hline
\end{tabular}


Tabla 5. Cobertura para la atención del pie diabético de acuerdo a el Catálogo Universal de Servicios de Salud, 2018

\begin{tabular}{|c|c|}
\hline Número 103 & $\begin{array}{l}\text { Diagnóstico y tratamiento de prediabetes y DM tipo } 2 \text { en el módulo de prevención y promoción de la salud. Está } \\
\text { enfocado al manejo médico de la enfermedad. }\end{array}$ \\
\hline Número 216 & $\begin{array}{l}\text { Tratamiento de úlcera y del pie diabético infectado con complicaciones circulatorias periféricas en el módulo de } \\
\text { urgencias médicas. Se proveen los insumos o medicamentos disponibles, que incluyen AAS, antibióticos de espectro } \\
\text { limitado, analgésicos, anestésicos locales, exámenes de laboratorio generales y una radiografía de dos posiciones (de } \\
\text { poca utilidad para el diagnóstico vascular). Incluye también el manejo en el servicio de urgencias, hospitalización y } \\
\text { consulta de medicina general y especialidad, pero no incluye terapia intensiva. }\end{array}$ \\
\hline Número 268 & $\begin{array}{l}\text { Amputación de miembro inferior secundaria a pie diabético con complicaciones circulatorias periféricas. Cubre } \\
\text { antibióticos de amplio espectro, heparinas y analgésicos de mayor potencia. Los auxiliares diagnósticos que se } \\
\text { incluyen son la angiografía de las extremidades, la radiografía de dos posiciones y el ultrasonido Doppler vascular } \\
\text { venoso y arterial. Su trazabilidad va desde el servicio de urgencias, la amputación por cirugía general, la } \\
\text { hospitalización y la terapia intensiva postoperatoria, así como la consulta de medicina general y especialidad para el } \\
\text { seguimiento. }\end{array}$ \\
\hline
\end{tabular}

AAS: ácido acetilsalicílico; DM: diabetes mellitus.

aunque mayormente enfocado en la prevención, cuenta con los resultados positivos del CAIPaDi y el servicio de heridas y estomas en el INCMNSZ.

\section{Seguro Popular, ahora Bienestar 42}

Brinda servicios de salud a personas sin seguro social de gastos médicos y tiene la capacidad de trabajar con distintos proveedores de servicios de salud tanto pública como privada en todos los estados de la República. Trata al $4.40 \%$ de la población diabética ${ }^{41}$.

En fechas recientes, en el Catálogo Universal de Servicios de Salud de 2018, de la Comisión Nacional de la Protección Social en Salud/Seguro Popular ${ }^{42}$, se cuenta con cobertura en los apartados para la atención del pie diabético descritos en la tabla 5 .

La actualización de los números 216 y 268 constituye un gran trabajo y complemento al programa Bienestar, ya que en versiones previas solo se contemplaban las amputaciones secundarias a pie diabético. De acuerdo con lo estipulado, es factible el diagnóstico vascular, pero aún queda por integrar la revascularización, para cuya planeación también toma importancia la angiotomografía computada. Es decir, que, a pesar de contar con antibióticos y medicamentos adyuvantes para mejorar el estado circulatorio previo a una amputación sin revascularización, aún es una maniobra incompleta, pues se condena al paciente a una amputación mayor, auna mortalidad incrementada y una rehabilitación compleja, con altos costos a la sociedad.

\section{Instituto Mexicano del Seguro Social}

Concentra al $44.9 \%$ de las hospitalizaciones por diabetes del país ${ }^{43}$ y al $37.64 \%$ de los tratados por diabetes ${ }^{41}$. Cubre tanto los medicamentos como los insumos necesarios para lograr la atención integral del pie diabético, en específico exámenes de laboratorio, rayos $\mathrm{X}$, electrocardiogramas, estancia hospitalaria, procedimientos quirúrgicos (debridaciones, aseos quirúrgicos, revascularizaciones, amputaciones menores y mayores) e interconsultas con especialidades. Asimismo, cuenta con un servicio de manejo de heridas y estomas, dedicado al cuidado de esta complicación ${ }^{43}$. Desde 1969 tiene programas de detección oportuna de diabetes (DiabetIMSS) que son aplicados por enfermeras y auxiliares especialistas en salud pública, y además se desarrollan continuamente normas que se difunden en cursos y talleres, a fin de consolidar la intervención de prevención de la $\mathrm{DM}^{44,45}$.

\section{Instituto de Seguridad y Servicios Sociales de LOS Trabajadores deL Estado}

Reporta una incidencia anual de 97,000 úlceras y 17,000 amputaciones. Esta institución atiende al 12.3\% de las hospitalizaciones por diabetes y al $5.6 \%$ de los tratados por $\mathrm{DM}^{41}$.

El programa de Manejo Integral de Diabetes por Etapas (MIDE) ${ }^{46}$ busca reducir la mortalidad por diabetes. Se centra en el paciente y busca innovar y mejorar la atención primaria, promover el empoderamiento y el autocuidado y la capacitación del equipo multidisciplinario y del paciente, que en un inicio es atendido por un médico familiar o por PrevenISSSTE, y son ellos quienes lo refieren al módulo MIDE, donde recibe atención multidisciplinaria. Si después de tres consultas subsecuentes en el año no se logra el control de la $\mathrm{HbA1c}$, se realiza la referencia a segundo nivel de atención. Los procedimientos del ISSSTE para el 
manejo de úlceras complejas y del pie diabético proponen dos vías principales, una quirúrgica y otra de medicina hiperbárica, a efecto de evitar amputaciones. La vía quirúrgica debe incluir el control metabólico y la interconsulta con el angiólogo y el cirujano vascular, quienes cuentan con distintos insumos para revascularizar, respectivos a cada centro hospitalario, en su mayoría de tercer nivel ${ }^{40,47}$.

La vía de medicina hiperbárica se realiza en el tercer nivel, lo cual limita la disposición de instalaciones. Se determina la dosis del tratamiento a aplicar, el tiempo de isopresión, el número de sesiones y su frecuencia. Por último, se hace una revaloración de la condición del paciente y de no haber mejoría se envía a revaloración multidisciplinaria ${ }^{40}$.

\section{Instituto de Seguridad y Servicios Sociales de los Trabajadores del Estado, la Secretaría de Marina, la Secretaría de la Defensa Nacional y Petróleos Mexicanos}

Estos servicios equivalen al $4.48 \%$ de la población con diabetes ${ }^{41}$. En ellos el tratamiento del pie diabético teóricamente se encuentra cubierto, sin embargo, no se cuenta con clínicas de atención integral, como las implementadas en los servicios de seguridad social ya explicados. El manejo actual del pie diabético se basa en lo que se propone en las Guías de práctica clínica para el manejo integral del pie diabético ${ }^{40}$.

\section{Cobertura en servicios de salud privados}

Cabe destacar que el $20.44 \%$ de la población con DM cubre los gastos en atención privada ${ }^{41}$, y que anteriormente no había disponibilidad de seguros de gastos médicos mayores para DM. Sin embargo, el Grupo AXA ofrece una excepción con el seguro de gastos médicos para diabéticos, en su programa "Protección Diabetes Flex Plus", el cual cubre a aquellos pacientes diagnosticados con DM2 en los últimos 5 años, siempre y cuando cumplan los siguientes requisitos: tener entre 28 y 60 años de edad, tener un IMC menor de 30 , sin otras enfermedades asociadas, como hipertensión, cáncer, antecedentes de infarto al miocardio, angina de pecho, cirrosis hepática, hepatitis $\mathrm{C} \circ \mathrm{VIH}, \mathrm{y}$ vivir en ciertas ciudades del país. A pesar de que el Grupo AXA acepta cubrir los gastos derivados de complicaciones, como la neuropatía, padecimientos renales, cardiovasculares, retinopatías, cetoacidosis, hipoglicemia, síndrome hiperglucémico, coma hiperosmolar no cetósico, insuficiencia venosa de miembros inferiores y cataratas, la atención del pie diabético no se encuentra contemplada. Por otra parte, la cobertura no es válida hasta pasados 36 meses de su contratación y tiene condiciones estrictas de renovación (mínimo de 3-4 consultas de monitoreo anual manteniendo una $\mathrm{HbA} 1 \mathrm{C}<7 \%$, un $\mathrm{IMC}<30$ y albuminuria negativa $)^{48}$. El resto de la atención en medio privado se realiza en forma directa con especialistas fuera de un centro integral de manejo.

\section{El pie diabético como enfermedad que causa gastos catastróficos}

Hemos discutido ampliamente el pie diabético es una enfermedad que provoca gastos catastróficos en el paciente, el núcleo familiar y su entorno. No se encuentra enlistada en la más reciente modificación de las enfermedades que ocasionan gastos catastróficos en $2018^{49}$. Esto pese a que en esta lista se incluyen enfermedades asociadas y agravadas por la DM, como infarto agudo de miocardio (IAM) y enfermedad vascular cerebral (EVC) e insuficiencia renal crónica. La importancia de su definición como tal es el establecimiento del procedimiento y los criterios a que se sujetará la misma para el estudio, análisis, elaboración y actualización de los protocolos técnicos en los que se incluyan los tratamientos, medicamentos y demás materiales asociados a los referidos gastos ${ }^{50} \mathrm{y}$, en consecuencia, al acceso al fondo de gastos catastróficos.

De acuerdo con el Reglamento Interior de la Comisión para Definir Tratamientos y Medicamentos Asociados a Enfermedades que Ocasionan Gastos Catastróficos ${ }^{50}$, el pie diabético cumple con los criterios solicitados para ser considerado una enfermedad que ocasiona gastos catastróficos, por lo cual es imperativo realizar su propuesta por una comisión formada de múltiples representantes de las autoridades hospitalarias, las universidades públicas, las academias y la Funsalud.

Una vez integrada esta comisión, se debe elaborar la solicitud con base en los lineamientos para la priorización de enfermedades e intervenciones que ocasionan gastos catastróficos, y en apego a la Clasificación Estadística Internacional de Enfermedades y Problemas relacionados con la Salud (CIE-10), definidos para el pie diabético, con sus múltiples afecciones, incluyendo la vascular, en los puntos E08.4, E08.5, E08.6 y E08.62 $2^{51}$. Entre los documentos necesarios se deben explicar las razones que motivan la solicitud, un análisis del peso de la enfermedad (AVISA, discapacidad), análisis de aceptabilidad social, evolución de la enfermedad y un estudio de impacto económico. Asimismo, 
deben incluirse todos los medicamentos y demás materiales asociados propuestos para gastos catastróficos dentro del Cuadro básico y el Catálogo de insumos del sector salud ${ }^{52}$ (Tabla 4).

\section{Conclusiones}

El pie diabético podría interpretarse como el resultado catastrófico de una desatención continua de la salud de un diabético en la que participan el paciente, el médico y el estado. Es necesario eliminar las barreras que impiden el manejo integral de esta desastrosa complicación en todas las instituciones que conforman el sistema de salud en México.

El tratamiento del pie diabético es un intento desesperado para conservar la calidad de vida que se extingue en el núcleo familiar y de alguna manera recuperar la felicidad. Su costo e impacto van más allá de lo que se percibe en las unidades hospitalarias y consultorios privados. La enfermedad no solo afecta al paciente, sino a la familia, que se ve obligada a dedicar parte de su vida a movilizar y auxiliar al paciente en sus actividades cotidianas, situación que deteriora la vida y economía de ambos actores. Quienes padecen úlceras complicadas y/o amputaciones representan costos desmesurados en la economía propia, en la de los empleadores, en la nacional y en los de la sociedad; por lo mismo el empoderamiento del paciente en el control de su enfermedad es fundamental en el éxito de cualquier intervención.

Las estrategias que se han implementado deben enfocarse en la prevención primaria, en el control de las complicaciones y en la promoción de la revascularización basada en la decisión de un equipo multidisciplinario en centros de atención integral, últimos que se perfilan como la herramienta con mejores resultados costo-beneficio comprobados. Los avances positivos en política de salud deben ser superiores a los intereses particulares y trascender y evolucionar en favor de la salud de los mexicanos, de acuerdo con la evidencia científica, la misma que debe ser difundida de manera oportuna y aprovechando la tecnología o los recursos disponibles. Esto demanda actualización, revisión y análisis continuo, así como la participación comprometida de todos los sectores, a fin de revertir la creciente pérdida de extremidades a causa del pie diabético.

\section{Agradecimientos}

A CONACYT por su apoyo a través del Sistema Nacional de Investigadores.

\section{Financiamiento}

Estímulo Convocatoria 2019 del Sistema Nacional de Investigadores CVU 402520

\section{Conflicto de intereses}

Los autores declaran que no existe conflicto de intereses.

\section{Responsabilidades éticas}

Protección de personas y animales. Los autores declaran que para esta investigación no se han realizado experimentos en seres humanos ni en animales.

Confidencialidad de los datos. Los autores declaran que en este artículo no aparecen datos de pacientes.

Derecho a la privacidad y consentimiento informado. Los autores declaran que en este artículo no aparecen datos de pacientes.

\section{Bibliografía}

1. Han Cho N. IDF Diabetes Atlas [Internet]. 8th ed. International Diabetes Federation; 2017. Disponible en: www.diabetesatlas.org

2. International Diabetes Federation; Sinclair A, Dunning T, Colagiuri S. IDF Clinical Practice Recommendations on the Diabetic Foot-2017. International Diabetes Federation. 2013.

3. Hernández M, Gutiérrez JP. Ensanut 2012. Diabetes mellitus: la urgencia de reforzar la respuesta en políticas públicas para su prevención y control. México, Instituto Nacional de Salud Pública. Disponible en: https:// ensanut.insp. $\mathrm{mx} /$ doctos/analiticosDiabetesMellitus.pdf

4. Boulton AJM. The diabetic foot: from art to science. The 18th Camillo Golgi lecture. Diabetología [Internet]. 2004; [citado el 10 de julio de 2019]; 47 (8). Disponible en: http://link.springer.com/10.1007/s00125-004-1463-y

5. Nehler MR, Coll JR, Hiatt WR, Regensteiner JG, Schnickel GT, Klenke WA, et al. Functional outcome in a contemporary series of major lower extremity amputations. J Vasc Surg. 2003;38(1):7-14

6. Híjar Medina M. Epidemiología de las amputaciones en México. Disponible en: Los amputados y su rehabilitación. Un reto para el Estado. México: Academia Nacional de Medicina; 2016.

7. Huseynova K, Sutradhar R, Booth GL, Huang A, Ray JG. Risk of contralateral lower limb amputation and death after initial lower limb amputation -a population-based study. Heliyon. 2018;4(10):e00836.

8. Internal Clinical Guidelines Team. Diabetic Foot Problems: Prevention and Management [Internet]. Londres, National Institute for Health and Care Excellence (reino Unido); 2015 [citado el 10 de julio de 2019]. (National Institute for Health and Care Excellence: Clinical Guidelines). Disponible en: www.ncbi.nlm.nih.gov/books/NBK338144/

9. Boulton AJ, Vileikyte L, Ragnarson-Tennvall G, Apelqvist J. The global burden of diabetic foot disease. The Lancet. 2005;366 (9498):1719-24.

10. Barraza Llorens V, Hernández Viveros C, Pico Guzmán FJ. Carga económica de la diabetes mellitus en México, 2013 [Internet]. México: Fundación Mexicana para la Salud ACE; 2015.

11. OCDE Stat. Organization for Economic Co-Operation and Development. Disponible en: https://stats.oecd.org/

12. Instituto Mexicano del Seguro Social. Datos de certificados de incapacidad y de dictámenes de invalidez. México: IMSS; 2013.

13. Montiel-Jarquín ÁJ, García Villaseñor A, Castillo Rodríguez C, Romero-Figueroa MS, Etchegaray-Morales I, García-Cano E, et al. Costes directos de atención médica del pie diabético en el segundo nivel de atención médica. Revista Chilena de Cirugía. 2017;69(2):118-23.

14. Lozano R, Gómez-Dantés H, Garrido-Latorre F, Jiménez-Corona A, Campuzano-Rincón JC, Franco-Marina F, et al. La carga de enfermedad, lesiones, factores de riesgo y desafíos para el sistema de salud en México. Salud Publica Mex. 2013;7:55(6):580. 
15. Arredondo A, Reyes G. Health Disparities from Economic Burden of Diabetes in Middle-income Countries: Evidence from México. PLoS One. 2013;8(7):e68443.

16. Ragnarson Tennvall G, Apelqvist J. Prevention of diabetes-related foot ulcers and amputations: a cost-utility analysis based on Markov mode simulations. Diabetología. 2001;44 (11):2077-087.

17. Yesil S, Akinci B, Bayraktar F, Havitcioglu H, Karabay O, Yapar N., et al. Reduction of Major Amputations after Starting a Multidisciplinary Diabetic Foot Care Team: Single Centre Experience from Turkey. Exp Clin Endocrinol Diabetes. 2009;117(07):345-9.

18. Larsson J, Apelqvist J, Agardh CD, Stenström A. Decreasing incidence of major amputation in diabetic patients: a consequence of a multidisciplinary foot care team approach? Diabet Med. 1995;12:770-6.

19. Ollendorf DA, Kotsanos JG, Wishner WJ, Friedman M, Cooper T, Bittoni $\mathrm{M}$, et al. Potential economic benefits of lower-extremity amputation prevention strategies in diabetes. Diabetes Care. 1998;21:1240-5.

20. Van Gils C, Wheeler L, Mellstrom M, Brinton E, Mason S, Wheeler C Amputation prevention by vascular surgery and podiatry collaboration in high-risk diabetic and nondiabetic patients. The Operation Desert Foot experience. Diabetes Care. 1999;22(5):678-83.

21. Driver V, Fabbi M, Lavery L, Gibbons G. The costs of diabetic foot: The economic case for the limb salvage team. J Vasc Surg. 2010;52(3):17S-22S.

22. Ortegon M, Redekop W, Niessen L. Cost-Effectiveness of Prevention and Treatment of the Diabetic Foot: A Markov analysis. Diabetes Care. 2004;27(4):901-7.

23. Peters E, Lavery L. Effectiveness of the Diabetic Foot Risk Classification System of the International Working Group on the Diabetic Foot. Diabetes Care. 2001;24 (8):1442-7.

24. G. Matricali; G. Dereymaeker; E. Muls; M. Flour y C. Mathieu. Economic aspects of diabetic foot care in a multidisciplinary setting: a review. Diabetes/Metabolism Research and Reviews. 2007; 23 (5): 339-347.

25. Secretaría de Salud, Gobierno de la República. Estrategia Nacional para la Prevención y Control del Sobrepeso, la Obesidad y la Diabetes. México; 2013. Disponible en: www.gob.mx/cms/uploads/attachment file/276108/estrategia sobrepeso diabetes obesidad.pdf

26. Instituto Nacional de Estadística Geografía e Informática. Mortalidad por causas de defunción. México; 2011. Disponible en: www. inegi.org.mx sistemas/temas/default.aspx?s=est\&c=17484

27. Instituto Nacional de Estadística Geografía e Informática. Características de las defunciones registradas en México durante 2017. México; 2018. Disponible: www.inegi.org.mx/contenidos/saladeprensa/boletines/2018/ EstSociodemo/DEFUNCIONES2017.pdf

28. Flores Ramírez J, Aguilar Rebolledo F. Diabetes mellitus y sus complicaciones. La epidemiología, las manifestaciones clínicas de la diabetes tipo 1 y 2. Diabetes gestacional. Parte 1. Plast Rest Neurol 2006;5 (2):139-51.

29. Presidencia de la República. Tres pilares de la Estrategia Nacional para un México sin Obesidad. México; 2013. Disponible en: www.presidencia. gob.mx/3-pilares-de-la-Estrategia-nacional-para-un-mexico-sin- obesidad/

30. Hernández-Jiménez S, García-Ulloa A, Bello-Chavolla O, Aguilar-Salinas C, Kershenobich-Stalnikowitz D. Long-term effectiveness of a type 2 diabetes comprehensive care program. The CAIPaDi model. Diabetes Res Clin Prac. 2019;151:128-37.

31. León-Mazón M, Araujo-Mendoza GJ, Linos-Vázquez ZZ; DiabetIMSS Eficacia del programa de educación en diabetes en los parámetros clínicos y bioquímicos. Revista Médica del Instituto Mexicano del Seguro Social. 2012;51(1):74-9

32. Rivera Dommarco JA, Hernández Ávila M, Aguilar Salinas CA, Vadillo Ortega F, Murayama Rendón C. Obesidad en México: recomendaciones para una política de Estado. México: UNAM. 2012:125-44.

33. Gutiérrez JP, Rivera-Dommarco J, Shamah-Levy T, Villalpando-Hernández S, Franco A, Cuevas-Nasu L, et al. Encuesta Nacional de Salud y Nutrición 2012. Resultados Nacionales. Cuernavaca, México: Instituto Nacional de Salud Pública; 2012.

34. Consejo Nacional de Salubridad General. México; 2015. Disponible en: www.csg.gob.mx/consejo/mision.html

35. Consejo de Salubridad General. Cuadro básico y Catálogo de medicamentos. México, Consejo de Salubridad General; 2017. Disponible en: www. csg.gob.mx/contenidos/priorizacion/cuadro-basico/med/catalogos.html
36. Consejo de Salubridad General. Cuadro básico y Catálogo de instrumental y equipo médico. México: Consejo de Salubridad General; 2017. Disponible en: www.csg.gob.mx/contenidos/priorizacion/cuadro-basico/iyem/ catalogos.html

37. Consejo de Salubridad General. Cuadro básico y Catálogo de material de curación. México: Consejo de Salubridad General; 2017. Disponible en: www.csg.gob.mx/contenidos/priorizacion/cuadro-basico/mc/catalogos.html

38. Consejo de Salubridad General. Cuadro básico y Catálogo de auxiliares de diagnóstico. México: Consejo de Salubridad General, 2017. Disponible en: www.csg.gob.mx/contenidos/priorizacion/cuadro-basico/aux diag/catalogos.html

39. Secretaría de Salud. Norma Oficial Mexicana NOM-015-SSA2-2010 para la prevención, tratamiento y control de la diabetes mellitus. México: Secretaría de Salud; 2010. Disponible en: www.dof.gob.mx/normasOficiales/4215/salud/salud.htm

40. Consejo de Salubridad General, Manejo integral del pie diabético. Manual de atención en adultos de segundo nivel de atención. Guía de práctica clínica. México, Cenetec, 2013. Disponible en: www.cenetec.salud.gob. $\mathrm{mx} /$ descargas/gpc/CatalogoMaestro/005_GPC_PieDiabetico/ SSA_005_08_GRR.pdf

41. Villalpando S, Rojas R, Shamah-Levy T, Ávila MA, Gaona B, De la Cruz V, et el. Prevalencia y distribución de la diabetes mellitus tipo 2 en población adulta mexicana. Una encuesta probabilística. Salud Pública Mex. 2010;52, supl. 1:S19-S26.

42. Seguro Popular. México: Seguro Popular; 2017. Disponible en: www. seguropopular.org

43. Montiel-Jarquín Á, García Villaseñor A, Castillo Rodríguez C, Romero-Figueroa M, Etchegaray-Morales I, García-Cano E, et al. Costes directos de atención médica del pie diabético en el segundo nivel de atención médica. Revista Chilena de Cirugía. 2017;69(2):118-23.

44. Velasco-Contreras ME. Evolución de la epidemia de diabetes mellitus tipo 2 en población derechohabiente del IMSS. Rev Med Inst Mex Seg Soc. 2016:54 (4):490-503.

45. Margot González L, Fernández Gárate JE, Hernández Cuesta IT, Torres Rojas NM, Cisneros González N, Rascón Pacheco RA, et al. Presentación: La carga de las enfermedades en el IMSS, 2010. México: IMSS, Dirección de Prestaciones Médica Publicación; 2012.

46. Dirección Médica ISSSTE. Introducción del programa de manejo integral de diabetes por etapas. (MIDE). México: Dirección Médica ISSSTE; 2011. Disponible en: http://sgm.issste.gob.mx/medica/diabetes/doctos/ Curso\%20de\%20Induccion\%20al\%20MIDE/Introduccion\%20al\%20programa\%20MIDE.pdf

47. Moya-Jiménez S, Morales-Ochoa YG, Serrano-Lozano JA. Incidence of major amputations secondary to diabetic foot prior and after endovascular revascularization. Rev Mex Angiol. 2020;48(2):11-6.

48. AXA Seguros. Gastos médicos mayores. Protección Diabetes Flex Plus ${ }^{\circledR}$ Condiciones generales. México: axa Seguros: 2018. Disponible en: https://axa.mx/documents/10928/13942158/GM CG_PROTECCIÓN+DIABETES+FLEX+PLUS.pdf/365365ef-812a-83e7-5567-7b0a523732f4

49. Diario Oficial de la Federación. Consejo General de Salubridad. Modificación a la lista actualizada de enfermeades que ocasionan gastos catastróficos. México: DOF; 2018. Disponible en: http://dof.gob.mx/nota_detalle.php? codigo $=5533136 \&$ fecha $=26 / 07 / 2018$

50. Diario Oficial de la Federación. Consejo General de Salubridad. Reglamento Interior de la Comisión para Definir Tratamientos y Medicamentos Asociados a Enfermedades que Ocasionan Gastos Catastróficos. México: DOF; 2017. Disponible en: www.csg.gob.mx/descargas/pdf/priorizacion/priorizacion/CSG-Reg Comision ER 2017.pdf

51. Organización Mundial de la Salud. Clasificación internacional de enfermedades. 10. ${ }^{a}$ ed. 1999. Disponible en: http://www.proteccioncivil.org/ catalogo/carpeta02/carpeta24/vademecum17/cie10/cie10E.htm

52. Consejo General de Salubridad. Lineamientos para la priorización de enfermedades e intervenciones que ocasionan gastos catastróficos. México: Consejo General de Salubridad, 2018. Disponible en: www.csg.gob. $\mathrm{mx} /$ contenidos/priorizacion/gastos-catastroficos/lineamientos.html 